\title{
ROC-BAC: ROLE AND CONTEXT BASED ACCESS CONTROL MODEL FOR THE HEALTHCARE ENVIRONMENT
}

\author{
Zanifa Omary \\ Department of Computer Science and Mathematics \\ The Institute of Finance Management, Tanzania
}

\begin{abstract}
This paper presents a new context-based access control model that allows healthcare professionals to bypass access rules in an accountable manner in case of unexpected (emergency) situation so as to provide continuity of care. The proposed RoC-BAC model is an extension of the conventional, policy-neutral, Role-Based Access Control (RBAC) model with two variables: contexts and obligations. In addition to being fine-grained, RoC-BAC model is dynamic, flexible and scalable and incorporates procedures for emergency access requests.
\end{abstract}

\section{KEYWORDS}

Access Control, Model, Role, Context, and Roc-Bac, Healthcare

\section{INTRODUCTION}

This paper aims at designing a new context-based access control model that allows healthcare workers to bypass access rules in an accountable manner in case of unexpected (emergency) situation. The proposed RoC-BAC model is an extension of the conventional, policy-neutral, Role-Based Access Control (RBAC) model with two variables: contexts and obligations. In addition to being fine-grained, RoC-BAC model is dynamic, flexible and scalable and incorporates procedures for emergency access requests. The reason behind a decision to incorporate health-related contexts into RBAC lies on accumulating evidence available on the existing literature. Many researchers in Ubiquitous Computing (Ahmed and Zhang, 2008; Covington et al., 2002; Tolone et al., 2005; Trnka and Cerny, 2016), where context has been thoroughly studied, point out that, when context is appropriately incorporated into an existing access control solution, the resulting model may improve security in critical applications. With this in mind, consider the significance of contexts in a military base as an example of improvement in security. Normally in a military base, a user is allowed to fire a missile if that user is assigned a certain role (for example, a top secret commander) and a user is in a specific high security location at a certain time only. Consequently, a missile can be fired only when it is in a certain location at a certain time. By adding location and temporal constraints in access decision, additional verification checks are henceforth included that must be satisfied before an individual user is granted access to fire a missile.

To ensure the same cross checking, Break-The-Glass (BTG) procedure has been introduced for the healthcare domain (Deshmukh and Croasdell, 2008; Petritsch, 2014; Preuveneers and Joosen, 2014; Souza et al., 2017) BTG is a significant and quick approach used in the domain to extend access privileges for a user who does not have access rights to a certain information to gain access when necessary (Marinovic et al., 2011; Petritsch, 2014; Petritsch and Brucker, 2009). The procedure draws its name from breaking-the-glass to activate a fire alarm. The healthcare systems that contains primary source data (information) for treatment must therefore develop, document, implement and test BTG procedures that would be used in the event when healthcare professionals require unexpected (emergency) access to those records. These systems must have a clearly stated and widely understood procedure for allowing access using an alternate or manual method. It should be noted that, the usage of emergency access rights facilitated by BTG approach needs to be 
documented for later audits and reviews. As discussed by NEMA et al., (2014), examples of infrequent situations that may require BTG and logging could be

1. When user experiences account problems such as forgotten username or password or both (after an extended absence or vacation), locked password (as a result of mistyping many times) and even no user account (e.g. a clinician from another organisation or a new clinician is assisting in facility during an emergency)

2. When there is an authentication problem including failure of the Central Authentication System (CAS) server, smart card or biometrics reader failure (that is, when a smart card reader or biometric is damaged), and

3. When there are authorization problems, including an emergency situation which thrusts an individual user into a role where he or she lacks sufficient access rights (for example, an administrative assistant is entering orders during emergency).

As acclaimed by Ferreira et al., (2009), another reason for an implementation of BTG procedure in healthcare is that access control policies are defined with "maximum freedom of access" and "maximum user responsibility" in order to ensure that nothing interferes with the delivery of care. To guarantee maximum freedom of access, information systems in healthcare must provide mechanisms for users to access requested information at any time, whenever the need arises. With maximum responsibility, systems in healthcare must provide a mechanism that offers additional information such as an alert making users requesting access to be aware that, the user is trying to access unauthorized information under normal healthcare settings or even through a call for help from other qualified people. Abstractly, this makes an individual user requesting such access to information responsible for what user is doing and may subsequently be held accountable. The system must also provide a mechanism that automatically notifies all responsible parties in relation to that access so that user's actions can be justified afterwards. To address this and other significant access control requirements for the healthcare domain, this paper discusses a new context-based access control model called Role and Context-Based Access Control (RoC-BAC), that allows healthcare professionals to bypass access rules in an accountable manner in case of unexpected (emergency) requests. With the proposed model, a new concept called health-related contexts is introduced. Section 2 discusses a new Role and Context-Based Access Control (RoC-BAC) model.

\section{ROLE AND CONTEXT-BASED ACCESS CONTROL (ROC-BAC) MODEL}

The Role and Context-Based Access Control (RoC-BAC) model is an extension of the traditional Role-Based Access Control model with the notion of health-related contexts and obligations. The model has been designed to allow healthcare professionals to bypass access rules in an accountable manner in case of unexpected (emergency) requests. This section presents the model in three parts: Section 2.1 presents a formal definition of components and relations of RoC-BAC model while Section 2.2 presents a formal RoC-BAC model. Finally, Section 2.3 presents a model augmented with obligations.

\subsection{RoC-BAC: Definition of Components and Relations}

This section defines components as well as relations in RoC-BAC model. To allow context-based access in healthcare, the RoC-BAC model incorporates health-related contexts in its access decisions so as to allow healthcare professionals to bypass access rules, and hence to support the continuity of care.

RoC-BAC model comprises of the following components:

i. Users (U) denote entities requesting access to objects. They may include human beings, processes, machines, networks or autonomous agents.

Mathematically, $\mathrm{U}=\left\{\mathrm{u}_{1}, \mathrm{u}_{2}: \ldots \mathrm{u}_{\mathrm{i}}\right\}$ denotes a set of users.

ii. Roles (R) represent organizational job functions with clear definition of inherent responsibility and authority. The concept of roles, as is used in this paper, is adopted from the RBAC model where 
users are assigned to roles (user roles) and roles are associated with permissions (permission roles). Furthermore, in RoC-BAC, roles represent a combination of user roles (UR) and context roles (CR), as such $\mathrm{R}=2(\mathrm{UR} \times \mathrm{CR})$

iii. An object (OBJ) is a passive entity that contains or receives information. A set of objects (OBJ) is represented mathematically as, $\mathrm{OBJ}=\left\{\mathrm{obj}_{1}, \mathrm{obj}_{2} \ldots \mathrm{obj}_{\mathrm{j}}\right\}$

iv. An operation (OPS) is an execution of a program-specific function that is invoked by the user. $\mathrm{OPS}=\left\{\mathrm{ops}_{1}, \mathrm{ops}_{2} \ldots \mathrm{ops}_{\mathrm{k}}\right\}$, denotes a set of operations recognized by the system.

v. Context (C) is defined as a set of variables that define a situation (Federenko et al., 2004). Contrary to traditional access control models that do not take into account contexts in determining whether access should be allowed or not, RoC-BAC offers a context-centric access control solution, that allow system administrators to define permissions based on the relevant contexts. Mathematically, $\mathrm{C}=\left\{\mathrm{c}_{1}, \mathrm{c}_{2} \ldots \mathrm{c}_{\mathrm{x}}\right\}$. For access control purposes, contexts in this paper are categorized in four groups:

- Subject Contexts (SC): define contexts that must be exercised by the subject in order to obtain access rights to an object or resource. In RoC-BAC, subject contexts are used to determine access rights for an entity requesting access privileges. They may include the subject's role, identity, credentials, name, organisation that the subject is affiliated with, activity, location, and task (Omary et al., 2011).

- Object Contexts (OC): An object is a passive entity that is acted upon by a subject. Similar to subjects, objects contain contexts that can be used to control their accesses. Object Contexts (OC) refer to any object-related information that can be used to characterize the situation in which the protected object was created and its current status, which is relevant for making an access control decision.

- Environment Contexts (EC): These contexts describe operational, technical, and even situational contexts at the time a transaction takes place. They may include current date and time, temperature, network's security level, air quality, light level, noise level. These factors are neither associated with a subject nor a resource, but may nevertheless be relevant in controlling access (Omary et al., 2011).

- Health-related Contexts (HC): these variables define the healthcare domain specific contexts that would be evaluated by an access control system during access decision. Some of the specific health-related contexts (based on the region when the service is offered) may include Gender-Based Violence (GBV), Female Genital Mutilation (FGM), non-surgical amputation of limbs for people with albinism, clashes between: pastoralist groups, pastoralist and farmers, among others. Even though the main aim of this paper is to extend the traditional RBAC model with health-related contexts; these four groups can further be used to define context roles in RoC-BAC, and thus extending the traditional RBAC model with contexts.

vi. User Roles (UR) represent a set of user roles. User role is similar to "role" or "subject role" as used in a traditional Role-Based Access Control (RBAC) model.

vii. Context Roles (CR) describe a set of context roles. It is used to capture relevant contexts that can be used in RoC-BAC policies. In addition to health-related context roles, RoC-BAC also contains time-based context roles, location-based context role and location and time-based context roles,

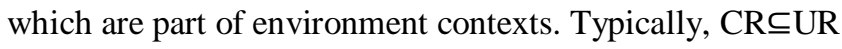

viii. Permission (P) is an approval to perform an operation on one or more objects. Permission $(\mathrm{P})=\left\{\mathrm{p}_{1}, \mathrm{p}_{2} \ldots \mathrm{p}_{\mathrm{w}}\right\}$, is a set of permissions in a system such that $\mathrm{P}=2{ }^{\text {OPS } \times \text { OBJ }}$. PermOperation: ops $(\mathrm{p}: \mathrm{P}) \rightarrow\{$ ops $\subseteq \mathrm{OPS}\}$, is a permission-to-operation mapping which 
gives a set of operations associated with a permission $p$. Permobject: obj $(p: P) \rightarrow\{$ obj $\subseteq$ OBJ\}, a permission-to-object mapping which gives a set of objects associated with permission $\mathrm{p}$.

ix. Session (S) represents a set of sessions. Usually, a role is activated for a user during each session. Activated Role (AR) is a mapping between user roles (UR) and Context Roles (CR).

The RoC-BAC model consists of several relations, including User Assignment (UA), Permission Assignment (PA) and Context Role Assignment (CRA). These relations define associations between users, user roles, permission roles and context roles.

- User Assignment (UA): $\mathrm{UA} \subseteq \mathrm{U} \times \mathrm{R}$, many-to-many mapping that assigns a user role to a user

- Context Role Assignment (CRA) is a mapping that assigns contexts to a user role, such that $\mathrm{CRA} \subseteq \mathrm{UR} \times \mathrm{C}$

- $\quad$ Permission Assignment (PA): $\mathrm{PA} \subseteq \mathrm{P} \times \mathrm{R}$, many-to-many mapping that assigns permissions to a role

- assigned_users (ur: UR) $\rightarrow 2^{\mathrm{U}}$, a mapping of a user "ur" onto a set of users. Formally assigned_users (ur) $=\{u \in U \mid(u, u r) \in U A=\{u \in U \mid(u, u r) \in U A\}$

- $\quad$ assigned_permissions $(r: R) \rightarrow 2^{\mathrm{P}}$, mapping of role " $r$ " onto a set of permissions. Formally, assigned permissions $=\{p \in$ PRMS $\mid(p, r) \in P A\}$

- User Sessions: user_sessions $(\mathrm{u}: \mathrm{U}) \rightarrow 2^{\mathrm{S}}$, a mapping of user u onto a set of sessions

- Session Roles: session_roles $(\mathrm{s}: \mathrm{S}) \rightarrow 2^{\mathrm{R}}$, mapping of session "s" onto a set of roles. Formally, session_roles $(s)=\left\{r \in R \rightarrow \mid\right.$ (user_sessions $\left.\left.\left(s_{i}\right), r\right) \in U A\right\}$

- $\quad$ avail_session_perms $(\mathrm{s}: \mathrm{S}) \rightarrow 2^{\mathrm{P}}$, denotes permissions available to a user in a session

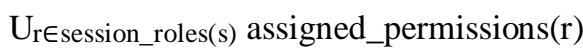

\subsection{Formal Definition}

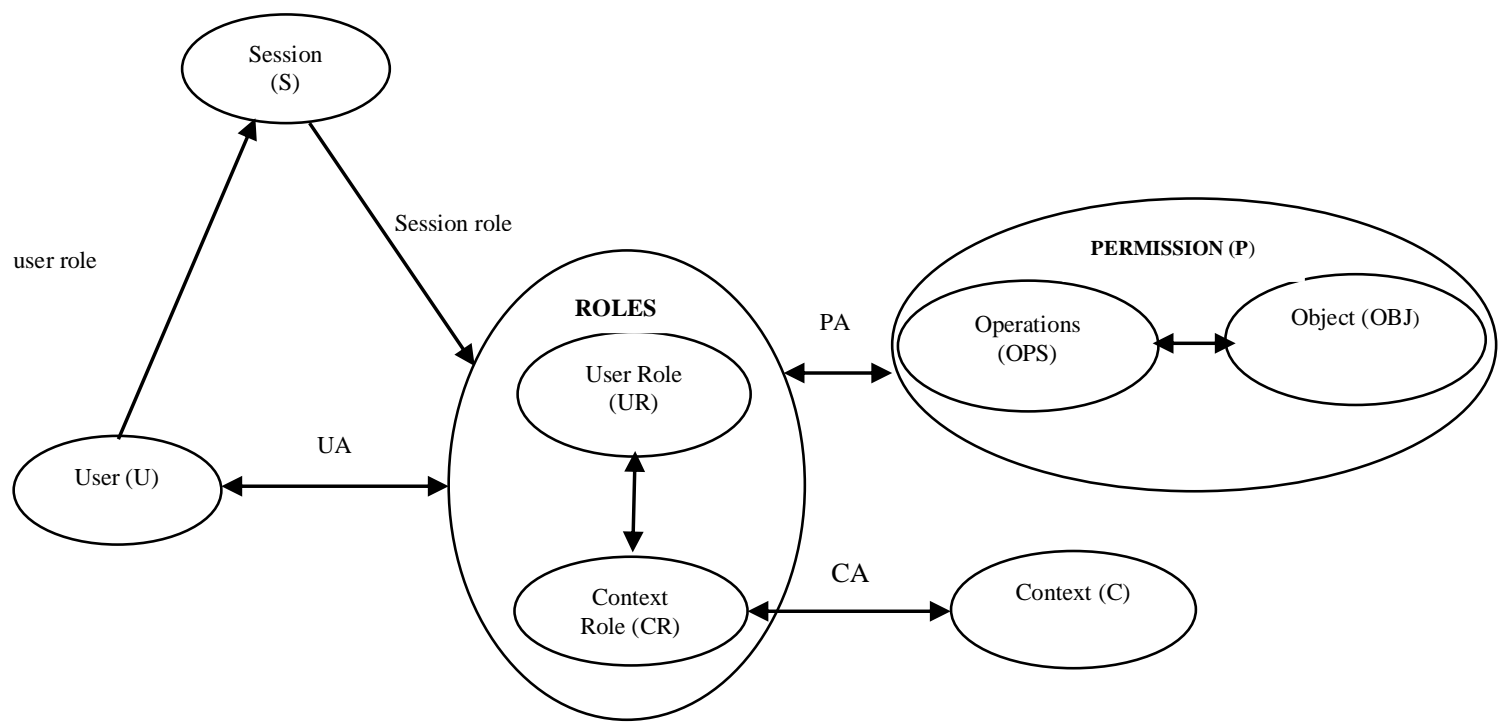

Figure 1. Role and Context Based Access Control (RoC-BAC) Model

The RoC-BAC model, presented in Figure 1, has the following components:

- $\quad \mathrm{U}, \mathrm{R}, \mathrm{C}, \mathrm{UR}, \mathrm{CR}, \mathrm{OPS}, \mathrm{OBJ}, \mathrm{P}$ and S (represents users, roles, context, user roles, context roles, operations, objects, permissions and sessions respectively)

- $\mathrm{UA} \subseteq \mathrm{U} \times \mathrm{R}$, a many-to-many mapping that assigns a user role to a user 
- assigned_users (ur: UR) $\rightarrow 2^{\mathrm{U}}$, a mapping of a user "ur" onto a set of users. Formally assigned_users (ur) $=\{\mathrm{u} \in \mathrm{U} \mid(\mathrm{u}, \mathrm{ur}) \in \mathrm{UA}\}$

- $\quad \mathrm{R} \subseteq 2(\mathrm{UR} \times \mathrm{CR})$, set of roles

- $\quad \mathrm{PA} \subseteq \mathrm{P} \times \mathrm{R}$, many-to-many permission to role assignment relation.

- assigned_permissions ( $\mathrm{r}: \mathrm{R}) \rightarrow 2^{\mathrm{P}}$, mapping of role " $\mathrm{r}$ " onto a set of permissions. Formally, assigned_permissions $=\{\mathrm{p} \in \mathrm{PRMS} \mid(\mathrm{p}, \mathrm{r}) \in \mathrm{PA}\}$

- user_sessions $(\mathrm{u}: \mathrm{U}) \rightarrow 2^{\mathrm{S}}$, a mapping of user $\mathrm{u}$ onto a set of sessions

- Session Roles: session_roles (s: S) $\rightarrow 2^{\mathrm{R}}$, mapping of session "s" onto a set of roles. Formally, session_roles $\left(\mathrm{s}_{\mathrm{i}}\right)=\left\{\mathrm{r} \in \mathrm{R} \rightarrow \mid\left(\right.\right.$ user_sessions $\left.\left.\left(\mathrm{s}_{\mathrm{i}}\right), \mathrm{r}\right) \in \mathrm{UA}\right\}$

\section{Definition 1: Context Role $(\mathrm{CR})=\left\{\mathrm{L}_{-} \mathrm{CR}, \mathrm{T} \_\mathrm{CR}, \mathrm{Hr} \_\mathrm{CR}\right\}$}

CR represents a set of Context Roles. They are used to capture context-relevant information for RoC-BAC access policies. In addition to health-related context roles, introduced in this paper through a notion of Health-related contexts, RoC-BAC model also supports the specification of Location-based Context Roles (L_CR) and Time-based Context Roles (T_CR). The context roles share similar characteristics with user roles, which are defined in a traditional RBAC model, such as role activation and deactivation.

Definition 1.1. L_CR (Location-based Context Roles)

Location-based Context Roles L_CR) is a pair (r, l) where $r$ denotes a user role name and 1 is the current position of a user role.

Definition 1.2. T_CR (Time-based Context Roles)

Similar to location, time-based Context Roles is a pair $(r$, time) where $r$ is a user role name and $t$ is the current time of a user role.

Definition 1.3. Hr_CR (Health-related Context Roles)

A Health-related Context Role (Hr_CR) is a pair (r, hr) where $\mathrm{r}$ is a user role name and $\mathrm{hr}$ represents a patient's current health condition that requires healthcare professionals' attention, thus referred as a health-related reason for access.

Definition 2: Authorizations

Location-based Authorizations are policies that define accesses that users may have over certain locations. While temporal authorizations limit periods of time during which an authorization is valid, health-related authorizations define accesses that users may have in case of an unexpected emergency. Formally, they are defined as follows:

Definition 2.1: L-Auth (Location Authorization)

A location authorization is a pair $((\mathrm{s}, \mathrm{r}), \mathrm{l})$ where

- $\mathrm{s}$ denotes a subject who requests authorization

- 1 is a location

- $\mathrm{r}$ is a role that subject $\mathrm{s}$ can be assigned when in location 1

A location authorization $((\mathrm{s}, \mathrm{r}), \mathrm{l})$ means that a subject $\mathrm{s}$ is authorized to gain role $\mathrm{r}$ when in location 1. ((Anne, Nurse), Ward) denotes that Anne has a role Nurse when she is in a hospital ward.

Definition 2.2: Ti_Auth (Temporal Authorization)

A temporal authorization is a quadruple $\left((\mathrm{s}, \mathrm{r}), \mathrm{t}\left(\left(\mathrm{t}_{\mathrm{s}}^{\mathrm{i}}, \mathrm{t}_{\mathrm{e}}^{\mathrm{i}}\right],\left(\mathrm{t}_{\mathrm{s}}^{\mathrm{o}}, \mathrm{t}_{\mathrm{e}}^{\mathrm{o}}\right]\right), \mathrm{n}\right)$ where

- Time interval [tis, tie] is an entry duration during which a subject can gain a role $\mathrm{r}$ to access object o

- Time interval $\left[\mathrm{t}_{\mathrm{s}}{ }_{\mathrm{s}}, \mathrm{t}_{\mathrm{e}}^{\mathrm{o}}\right]$ denotes an exit duration during which a subject $\mathrm{s}$ will revoke a role such that $\mathrm{t}_{\mathrm{s}}^{\mathrm{o}} \geq \mathrm{t}_{\mathrm{s}}^{\mathrm{i}}$ and $\mathrm{t}_{\mathrm{e} \geq}^{\mathrm{o}} \mathrm{t}_{\mathrm{e}}^{\mathrm{i}}$

- $\mathrm{n}$ is the number of accesses that subject $\mathrm{s}$ can exercise $\mathrm{n} \in[1, \infty)$ 
A temporal authorization ((Anne, Nurse), $(8,17],(17,8], 50)$ means Anne is allowed to activate role Nurse between $(8: 00,17: 00)$ and a role will be revoked fifty times during (17:00, 8:00) interval.

Definition 2.3: LoT_Auth (Spatial Temporal Authorization)

RoC-BAC also specifies spatial temporal authorization where policies contain both location and time-

related information. A spatial temporal authorization is defined as $\left((\mathrm{s}, \mathrm{r}), \mathrm{ops}, \mathrm{obj}, \mathrm{t}\left(\left(\mathrm{t}^{\mathrm{i}} \mathrm{s}, \mathrm{t}^{\mathrm{i}} \mathrm{e}\right),\left(\mathrm{t}^{\mathrm{O}} \mathrm{s}\right.\right.\right.$, $\left.\mathrm{t}^{\mathrm{O}} \mathrm{e}, \mathrm{l}\right)$ where

- $\quad$ s, r, ops and obj represent subject, role, operation and object respectively

- 1 is a location

- A spatial temporal authorization ( $(s, r)$, ops, obj, ftime, etime, l) semantically means a subject $\mathrm{s}$ with role $\mathrm{r}$ can perform operation ops on object obj under temporal condition ftime and etime and location condition 1.

Definition 2.4: Hr_Auth (Health-related Authorizations)

A health-related authorization is a pair $((\mathrm{s}, \mathrm{r}), \mathrm{hr})$ where

- $\quad \mathrm{s}$ represents a subject who requests an authorization and

- $r$ represents a role which s can activate

- $\mathrm{hr}$ is a health-related context that subject s can specify to access records of the patient

A health-related authorization ((Anne, Nurse), FGM) means Anne has a role Nurse but can also activate contextual role FGM when there is a new case to be attended in a respective health facility.

Definition 3: RoC-BACA (RoC-BAC Authorization)

RoC-BAC authorization is defined as RoC-BACA (Hr-Auth (L_Auth (T_Auth))) where

- L_Auth denotes location authorization

- T_Auth denotes temporal authorization

- Hr_Auth denotes health-related authorization.

Definition 4: RoC-BAC Authorization rule

The RoC-BAC authorization rule is defined by the tetrad (s, R_Auth, obj, m)

- $\quad \mathrm{S}$ denotes a subject (user) requesting access

- R_Auth is a RoC-BAC Authorization

- Obj is an authorized object

- M represents an access mode which can either be read or write

\subsection{Augmenting Obligations}

One limitation of the traditional access control mechanisms is their inability to provide users with obligations as a result of executing permitted action. This major weakness may result in the compromise of integrity or security of the system as system integrity, for instance, requires that certain action(s) performed in a system should always be followed by other action(s) within a limited time frame (Minsky \& Lockman, 1985). The RoC-BAC model is, therefore, augmented with obligations that are often imposed by operational rules, modern corporation regulations or privacy laws. Obligations specify actions that must be fulfilled in conjunction with an authorization decision (permit or deny) when an access rule is triggered to maintain accountability. This section presents how RoC-BAC model could be augmented with obligations, as shown in Figure 2 in order to maintain accountability in the system. Section 3 presents related work.

The following four enhancements were applied to the formal RoC-BAC model:

1. A new basic element called OBGS representing a set of valid obligations is introduced. In this paper, obligation is defined as task(s) that must be fulfilled by the system, and they are associated with permissions allocated to roles. They are categorized as pre-obligations, post-obligations, 
conditional obligations or repeating obligations. In this regard, the RoC-BAC model, which has been designed for the healthcare domain, is augmented with post-obligations to allow for healthcare professionals to access medical records in time, and thus ensure the continuity of care.

2. A new relation called Obligation Permission (OPRMS) is introduced. It represents a relation between permissions $(\mathrm{P})$ and obligations $(\mathrm{OBGS})$, such that OPRMS $\subseteq \mathrm{P} \times 2 \mathrm{OBG}$. For oprms $=(\mathrm{prms}$, obgs) $\epsilon$ OPRMS, means oprm is an obligation augmented permission which specifies if permission prm is exercised, a set of obligations obg should be fulfilled.

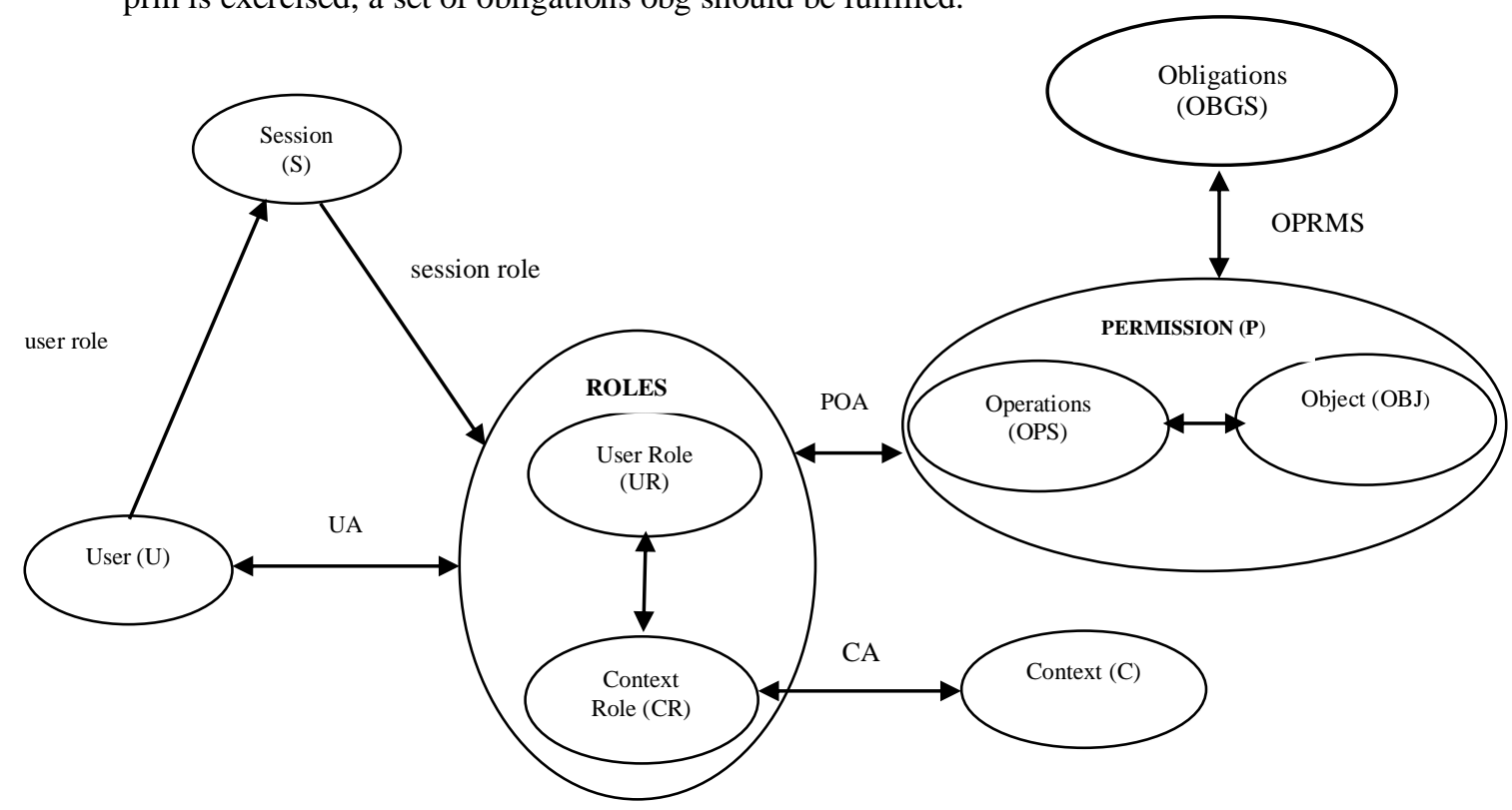

Figure 2. An augmentation of RoC-BAC model with obligations

3. Permission Assignment (PA) relation is modified and a new relation called Permission-Obligation Assignment (POA) is defined. Formally, POA $\subseteq$ ROLES $\times$ OPRMS. For $p=(r$, oprms $) \in P A$, it means role $\mathrm{r}$ is allocated with obligation augmented permission oprm.

4. To retrieve obligations with authorization decisions, Check Access function is modified into (Zhao et al., 2007)

CheckAccess: SESSIONS, OPS, OBJ $\rightarrow$ BOOL $\times 2^{\mathrm{OBG}}$

The possible combination between authorization elements and results from CheckAccess function are as follows:

- $\quad($ FALSE, $\varnothing) \rightarrow$ DENY access to a resource

- $\quad($ FALSE, 2OBG) $\rightarrow$ DENY access to a resource AND perform obligations on DENY

- $\quad($ TRUE, $\emptyset) \rightarrow$ GRANT access to a resource

- $\quad($ TRUE, 2OBG) $\rightarrow$ GRANT access to a resource and perform obligations on GRANT

\section{RELATED WORK}

Role-Based Access Control (RBAC) is a well-established access control model with widely-recognized advantages; and it is supported by a large number of software products and platforms (Sandhu, 1996), (Ferraiolo et al., 1995, 2001; Lodderstedt et al., 2002). The model addresses access control requirements of commercial organizations through an introduction of the role concept (Ferraiolo et al., 2003; Hu et al., 2006; Sandhu, 1996). In RBAC, permissions are attached to roles and users must be assigned to roles to get the permissions. A role represents a specific task competency, such as that of a medical doctor or lecturer, or it can embody the authority and responsibility (Sandhu, 1996). Roles in RBAC define both specific individuals allowed to access resources as well as the extent to which those resources can be accessed. Furthermore, 
permissions determine what operations can be carried out on resources under access control. A user must establish a session to activate a subset of roles to which the user is assigned. Each user can activate multiple sessions; however, each session is associated with only one user. The operations that a user can perform in a session depends on the roles activated in that session and permissions associated with those roles. The RBAC model supports roles hierarchy that define an inheritance relationship between roles. To prevent conflict of interest that may arise in an organisation, RBAC allows the specification of static and dynamic separation of duty constraints. Several works exist in the literature that improves RBAC functionality for different application domains. This section discusses the research works that are closely related to the access control model proposed in this paper, and particularly how RBAC has been extended with different concepts or notions to make the overall approach "context-based" or "context-aware".

As part of RBAC's extensions, Sampemane et al., (2002) introduced a new access control model for active spaces. The authors define an active space as "a computing environment that integrates physical spaces and embedded computing software and hardware entities. The active space allows interactive exchange of information between the user and the space". Environmental aspects are adopted into the access control model for active spaces, and the space roles are introduced into the implementation of the access control model based on RBAC. The proposed model supports specification of both Discretionary Access Control (DAC) and Mandatory Access Control (MAC) policies. While DAC policies allow users to create and update security policies for their devices, the MAC policies allow system administrators to maintain the access matrix. In addition to Sampemane et al., (2002), a development infrastructure for access spaces has been proposed by other researchers including Cerqueira et al., (2001).

(Covington et al., 2001) introduced a Generalized RBAC (GRBAC) model to help control access to private information and resources in ubiquitous computing applications. In fact, the GRBAC model extends the traditional RBAC model by incorporating the notion of object and environment roles. Even though environment roles differ from the subject roles in RBAC but they do have similar properties, including: role activation, role hierarchy and separation of duty. In an access control framework enabled by environment roles, each element of permission assignment is associated with a set of environment roles, and environment roles are activated according to changing conditions specified in environmental conditions. In this way, environmental properties such as location and time are introduced to the access control framework. Temporal Role-Based Access Control (TRBAC) is another extension of RBAC, proposed by (Bertino et al., 2001). This model adds a time dimension to the RBAC model whereas its authors introduce the concept of role enabling and disabling. The temporal constraints in the model determine when the roles can be enabled or disabled. A role can be activated only if it has been enabled. (Joshi et al., 2005) extend the TRBAC model by proposing the Generalized Temporal Role-Based Access Control (GTRBAC) model. In this work the authors introduce the concept of time-based role hierarchy and time-based separation of duty.

Several other researchers have also extended RBAC to incorporate spatial information. To cope with spatial requirements, Hansen and Oleshchuk, (2003) extend the conventional RBAC model and propose Spatial Role-Based Access Control (SRBAC) model. The SRBAC model utilizes location in its security policy definitions. Another important work in this regard is GEO-RBAC model proposed by Damiani et al., (2007), which is probably the most expressive location-based access control model. The role activation is based on the location of the user. Consider the following as a usage example of GEO-RBAC in a real world where a user can acquire a role of a patient when in a hospital grounds and a role of a teacher when in a school compound. The GEO-RBAC model supports the notion of role hierarchies but does not deal with separation of duties. Another work incorporating spatial information is by Ray et al., (2006). In this work, authors analyze how each component of a traditional RBAC is influenced by location. The authors define their model using Z specification language. The Location-Based Access Control has been addressed in some other works not pertaining to RBAC including (Leonhardt and Magee, 1998), (Hengartner and Steenkiste, 2004), and (Ray et al., 2006)

There are also other works that incorporate both location and time in to the traditional RBAC. The research work by Chandran and Joshi, (2005) combines the main features of GTRBAC and GEO-RBAC. In their LoT-RBAC model, role is enabled by time constraints. A user can activate a role if such a role is enabled and an individual user satisfies location constraints associated with role activation. Another feature worth mentioning from their work is that, when a role is activated all permissions associated with such a role can be invoked. Kumar and Newman, (2006) proposed the Spatial Temporal Role-Based Access Control model that is suitable for pervasive computing applications. With their access control model, the authors show the association of each component of the conventional RBAC model with spatial-temporal information. 
In addition to location and time, the traditional Role-Based Access Control (RBAC) model has also been extended using purpose information. $\mathrm{Ni}$ et al., (2010) proposed a purpose-based access control model for privacy protection. Their proposed model is based on the notion of purpose roles through which purpose is categorized into intended purposes and access purposes. In addition to role attributes and system attributes, Ni et al., (2010) also incorporate purpose hierarchy into their model. RBAC can also be extended by the notion of trust. Bhatti et al., (2005) proposed a trust-enhanced version of their XML-based access control (X-RBAC) framework for web services. The proposed model, which is an extension of RBAC with trust and contexts, incorporates context-based access control. Chakraborty and Ray, (2006) propose a trust-based access control model called TrustBAC. The model extends the conventional role-based access control model with the notion of trust levels. Users are assigned to trust levels instead of roles based on a number of factors, including user credentials, user behavior history, and user recommendation, and trust levels are assigned to roles which are assigned to permissions. Baracaldo et al., (2015) propose a Geo-Social RBAC, which is a new role based access control model that allows the inclusion of geo-social constraints as part of the access control policy. Besides capturing the locations of a user requesting access and her social connections, geo-social includes cardinality constraints that dictate how many people related by a particular social relation need to be present in the required locations at the time of an access

\section{CONCLUSION}

Despite a large number of contributions from various access control models in the existing literature, majority of these models do not deal with vital privacy-aware elements, such as obligations and complex conditions while providing access for unexpected situations, which form an essential part of privacy protection. With this limitation, this paper argues that, to gain full advantage of contexts in an access control solution, an access control solution should incorporate context conditions that are specific to the application domain. In this case, RoC-BAC model has been designed by extending the traditional Role-Based Access Control (RBAC) model with the notion of health-related contexts, from the healthcare domain.

\section{REFERENCES}

Ahmed, A., Zhang, N., 2008. A context-risk-aware access control model for ubiquitous environments. Presented at the 2008 International Multiconference on Computer Science and Information Technology, IEEE, pp. 775-782.

Baracaldo, N., Palanisamy, B., Joshi, J., 2015. Geo-social-rbac: A location-based socially aware access control framework. Presented at the International Conference on Network and System Security, Springer, pp. 501-509.

Bertino, E., Bonatti, P.A., Ferrari, E., 2001. TRBAC: A Temporal Role-based Access Control Model. ACM Trans Inf Syst Secur 4.

Bhatti, R., Bertino, E., Ghafoor, A., 2005. A trust-based context-aware access control model for web-services. Distrib. Parallel Databases 18, 83-105.

Cerqueira, R., Hess, C.K., Román, M., Campbell, R.H., 2001. Gaia: A development infrastructure for active spaces, in: Workshop on Application Models and Programming Tools for Ubiquitous Computing (Held in Conjunction with the UBICOMP 2001). p. 11.

Chakraborty, S., Ray, I., 2006. TrustBAC: integrating trust relationships into the RBAC model for access control in open systems, in: Proceedings of the Eleventh ACM Symposium on Access Control Models and Technologies. ACM, pp. 49-58.

Chandran, S.M., Joshi, J.B., 2005. LoT-RBAC: a location and time-based RBAC model, in: International Conference on Web Information Systems Engineering. Springer, pp. 361-375.

Covington, M.J., Fogla, P., Zhan, Z., Ahamad, M., 2002. A context-aware security architecture for emerging applications. Presented at the 18th Annual Computer Security Applications Conference, 2002. Proceedings., IEEE, pp. 249-258.

Covington, M.J., Long, W., Srinivasan, S., Dev, A.K., Ahmad, M., Abowd, G.D., 2001. Securing context-aware applications using environment roles, in: Proceedings of the Sixth ACM Symposium on Access Control Models and Technologies. ACM, pp. 10-20.

Damiani, M.L., Bertino, E., Catania, B., Perlasca, P., 2007. GEO-RBAC: a spatially aware RBAC. ACM Trans. Inf. Syst. Secur. TISSEC 10. 
Deshmukh, P., Croasdell, D., 2008. HIPAA: Privacy and security in health care networks, in: Information Security and Ethics: Concepts, Methodologies, Tools, and Applications. IGI Global, pp. 2770-2781.

Ferraiolo, D., Cugini, J., Kuhn, D.R., 1995. Role-based access control (RBAC): Features and motivations, in: Proceedings of 11th Annual Computer Security Application Conference. pp. 241-48.

Ferraiolo, D., Kuhn, D., Chandramouli, R., 2003. Role-Based Access Control. Artech House Publishers.

Ferraiolo, D.F., Sandhu, R., Gavrila, S., Kuhn, D.R., Chandramouli, R., 2001. Proposed NIST standard for role-based access control. ACM Trans. Inf. Syst. Secur. TISSEC 4, 224-274.

Ferreira, A., Chadwick, D., Farinha, P., Correia, R., Zao, G., Chilro, R., Antunes, L., 2009. How to securely break into RBAC: the BTG-RBAC model, in: Computer Security Applications Conference, 2009. ACSAC'09. Annual. IEEE, pp. 23-31.

Hansen, F., Oleshchuk, V., 2003. Spatial Role-Based Access Control Model for Wireless Networks, in: VTC 2003-Fall IEEE 58th Vehicular Technology Conference. pp. 2093-2097.

Hengartner, U., Steenkiste, P., 2004. Implementing access control to people location information, in: Proceedings of the Ninth ACM Symposium on Access Control Models and Technologies. ACM, pp. 11-20.

Hu, V., Ferraiolo, D., Kuhn, D., 2006. Assessment of Access Control Systems (No. NIST 7316), US Department of Commerce. National Institute of Standards and Technology.

Joshi, J.B., Bertino, E., Latif, U., Ghafoor, A., 2005. A Generalized Temporal Role-Based Access Control Model. IEEEE Trans. Knowl. Data Eng. 17, 2-23.

Kumar, M., Newman, R.E., 2006. STRBAC-An approach towards spatio-temporal role-based access control. Commun. Netw. Inf. Secur. 155.

Leonhardt, U., Magee, J., 1998. Security considerations for a distributed location service. J. Netw. Syst. Manag. 6, 51-70.

Lodderstedt, T., Basin, D., Doser, J., 2002. SecureUML: A UML-based modeling language for model-driven security. Presented at the International Conference on the Unified Modeling Language, Springer, pp. 426-441.

Marinovic, S., Craven, R., Ma, J., Dulay, N., 2011. Rumpole: a flexible break-glass access control model, in: Proceedings of the 16th ACM Symposium on Access Control Models and Technologies. ACM, pp. 73-82.

National Electrical Manufacturers Association, European Coordination Committee of the Radiological and Electromedical Industry, Japan Industries Association of Radiological Systems, 2014. Break-Glass - An Approach to Granting Emergency Access to Healthcare Systems. NEMA/COCIR/JIRA Security and Privacy Committee (SPC).

Ni, Q., Bertino, E., Lobo, J., Brodie, C., Karat, C.-M., Karat, J., Trombeta, A., 2010. Privacy-aware role-based access control. ACM Trans. Inf. Syst. Secur. TISSEC 13, 24.

Petritsch, H., 2014. A generic break-glass model, in: Break-Glass. Springer, pp. 37-50.

Petritsch, H., Brucker, A.D., 2009. Extending access control models with break-glass.

Preuveneers, D., Joosen, W., 2014. Federated Privileged Identity Management for Break-the-Glass: A Case Study with OpenAM. Presented at the PAHI, pp. 37-52.

Ray, I., Kumar, M., Yu, L., 2006. LRBAC: a location-aware role-based access control model, in: International Conference on Information Systems Security. Springer, pp. 147-161.

Sampemane, G., Naldurg, P., Campbell, R.H., 2002. Access Control for Active Spaces. IEEE, p. 343.

Sandhu, R., 1996. Access Control: The Neglected Frontier. Inf. Secur. Priv. Springer 219-227.

Souza, S., Gonçalves, R., Leonova, E., Puttini, R., Nascimento, A., 2017. Privacy-ensuring electronic health records in the cloud. Concurr. Comput. Pract. Exp. 29, e4045.

Tolone, W., Ahn, G.-J., Pai, T., Hong, S.-P., 2005. Access control in collaborative systems. ACM Comput. Surv. CSUR 37, 29-41.

Trnka, M., Cerny, T., 2016. On security level usage in context-aware role-based access control. Presented at the Proceedings of the 31st Annual ACM Symposium on Applied Computing, ACM, pp. 1192-1195.

Zhao, G., Chadwick, D., Otenko, S., 2007. Obligations for role based access control, in: Advanced Information Networking and Applications Workshops, 2007, AINAW’07. 21st International Conference On. IEEE, pp. 424-431. 